\title{
Surgical Pain Management: A Complete Guide to Implantable and Interventional Pain Therapies
}

\author{
Sanjeet Narang, Alison Weisheipl, Edgar L. Ross (editors). Oxford University Press, 2016. \\ ISBN 978-0-199377-37-4
}

\author{
Collin Clarke, MD (B)
}

Received: 29 July 2016/Revised: 3 August 2016/Accepted: 11 August 2016/Published online: 24 August 2016

(c) Canadian Anesthesiologists' Society 2016

One in five North Americans suffer from moderate to severe chronic pain, which is becoming a significant socioeconomic burden. Furthermore, as the population ages, it is anticipated that this proportion will grow. Accordingly, these patients and their physicians are seeking more sophisticated management strategies to address intractable pain. Academic and non-academic practices alike have been developing pain management programs that include an increasing array of surgical and intervention options for managing chronic pain.

With the advances in surgical approaches, many texts and reference materials are becoming available for the provider. Few texts, however, offer in-depth, comprehensive coverage of this topic. Narang et al.'s Surgical Pain Management: A Complete Guide to Implantable and Interventional Therapies - which includes topics that range from how to design and implement a surgical/interventional program to tips and strategies for managing complications and difficult patients - provides indispensable knowledge and experience with pain management from providers from one of the foremost academic centers in North America.

The text is arranged into three major sections. The first describes the foundation of a successful surgical/ interventional pain management team. This section is a "how to" guide on the development of such a service, outlining the key individuals required for efficient patient management as well as those who provide psychological assessments and patient education. For the readers who provide anesthetic care to chronic pain patients, this section

C. Clarke, MD (ه)

Western University, London, ON, Canada

e-mail: cclarke@uwo.ca contains an excellent review of the anesthetic considerations for these patients and the surgeries/ procedures they may be receiving. The strengths of the section are the small, soft features that are often overlooked by other texts, such as how to field patients' telephone calls effectively and tips for improving patient communication. The included case discussion on psychological patient assessment solidifies the learning in this chapter, especially as it is a topic not well covered in many anesthesia education programs. For those initiating a pain service or looking to improve their existing service, the authors give examples of standardized forms, including order forms for intrathecal drug sets and operative booking orders.

The second section thoroughly covers the vast majority of advanced interventional and surgical pain management procedures that are performed today, ranging from minimally invasive treatments for spinal stenosis to neuromodulation technologies. That said, if one is looking for reference material on the more commonly performed interventional procedures, such as epidural injection therapy and medial branch ablation, they will not be found in this text. Each topic is arranged in a standardized fashion, discussing preoperative selection and preparation, intraoperative performance and considerations, and finally postoperative concerns. The conclusion of the majority of the chapters includes a selection of case examples. These cases offer a wealth of clinical pearls along with the management of several unique, challenging patients. Professionals with years of acquired knowledge and expertise outline their management choices. The strength of this section is the review of evidence in support of the procedures and thorough, open discussions of studies that may lack appropriate power. Furthermore, when one of the authors 
is giving his or her expert opinion (i.e., in the absence of adequate evidence), it is readily disclosed, giving a transparency to indications while at the same time helping providers develop a strategy for treatment implementation. One drawback of this section is that a few chapters are somewhat sparse in their details on the technical performance of the procedures.

The final section is a series of well organized and constructed appendices. The appendices are succinct yet contain a wealth of useful information. They serve as a quick reference to a variety of important topics including, but not limited to, suturing, infection prophylaxis, procedural dictations, and cerebrospinal fluid pharmacotherapy. This section would benefit learners and the experienced practitioner alike.

This 408-page text effectively compiles the experience of 43 health care providers from the field of pain management into a single resource. The conciseness of this text makes it easy to reference. In addition, with its compact size and light weight, it could easily travel in a laptop bag or small backpack. The inclusion of case studies and the multiple forms for provision of care, not seen in other texts, serves to elevate its functional value. Although these technologies are part of a comprehensive pain management strategy, one cannot neglect the fact that this field is ever evolving. Even if the reader chooses not to incorporate these techniques into a practice, this text highlights what is available to patients who have been unable to find relief with conservative strategies.

Surgical Pain Management: A Complete Guide to Implantable and Interventional Therapies is a wellconstructed, easy to read reference guide to an everexpanding field of practice. The information compiled in this text would serve both the advanced learner, such as senior residents in anesthesia or pain medicine, and active practitioners looking to expand or improve their practice. Finally, this text contains useful administrative and organizational information that can help senior leaders of a pain management program improve the structure and function of a program.

Conflicts of interest None declared.

Editorial responsibility This submission was handled by Dr. Hilary P. Grocott, Editor-in-Chief, Canadian Journal of Anesthesia. 